\title{
Sexual Risk-Taking Behaviors, Gambling, and Heavy Drinking Among U.S. College Athletes
}

\author{
Jiun-Hau Huang · Durand F. Jacobs • \\ Jeffrey L. Derevensky
}

Received: 13 September 2007 / Revised: 16 February 2009 / Accepted: 7 June 2009

(C) Springer Science+Business Media, LLC 2009

\begin{abstract}
The purpose of this study was to empirically examine the prevalence patterns of sexual risk-taking behaviors (i.e., unprotected sex and having multiple sex partners) in relation to levels of gambling problems and heavy episodic drinking (HED) status among U.S. college athletes. Data from a representative national sample of 20,739 U.S. college athletes were derived from the first National Collegiate Athletic Association national survey of problem gambling and healthrisk behaviors. Among college athletes who were sexually active during the past year, males reported significantly higher prevalence of unprotected sex $(10.2 \%)$ and multiple sex partners (14.6\%) than females (7.9\% and 9.3\%, respectively). Using the DSM-IVGambling Screen classification, as the level of gambling severity increased, the prevalence of sexual risktaking behaviors also increased among female athletes, but decreased among male athletes. As regards the effect of heavy drinking, while both male and female HED athletes reported elevated sexual risk-taking, the effect of HED was twice as large in females as in males. It is important to note that the definitions of sexual risk behaviors in this study took into
\end{abstract}

J.-H. Huang $(\bowtie)$

Institute of Health Policy and Management, College of Public

Health, National Taiwan University, 17 Xu-Zhou Road, Taipei

10055, Taiwan

e-mail: jhuang@ntu.edu.tw

J.-H. Huang · J. L. Derevensky

International Centre for Youth Gambling Problems and High-Risk

Behaviors, McGill University, Montreal, QC, Canada

J.-H. Huang · J. L. Derevensky

Department of Educational and Counselling Psychology,

McGill University, Montreal, QC, Canada

D. F. Jacobs

Department of Psychiatry, Loma Linda University School

of Medicine, Loma Linda, CA, USA account committed sexual relationship status; hence, the results of this study need to be interpreted with the refined sexual risk measures in mind. Further investigations are warranted to help us better understand and explicate the interrelationships of sexual risk-taking behaviors, gambling, and heavy drinking among these college athletes. Findings from this exploratory study suggest new directions for future research and practice and also highlight the importance of a more inclusive multicomponent approach to address these co-occurring youth risk behaviors.

Keywords Unprotected sex - Multiple sex partners . Gambling · Heavy episodic drinking · College athletes . Gender

\section{Introduction}

STD/HIV Incidence and Sexual Risk Behaviors Among Youth

Sexually transmitted diseases (STDs) remain a major public health issue in the U.S., especially among youth. In a recent study which provided the first extensive national estimates for the incidence and prevalence of STDs among adolescents and young adults in the U.S., epidemiologists at the Centers for Disease Control and Prevention (CDC) estimated that there are approximately 18.9 million new cases of STDs each year, and that nearly half of these new cases (9.1 million) occur among young people aged 15-24, although they represent just $25 \%$ of the sexually experienced population (Weinstock, Berman, \& Cates, 2004). In addition to the physical and psychological consequences, these 9.1 million cases of STDs, including HIV infections, are associated with a lifetime direct medical cost of 6.5 billion dollars (Chesson, Blandford, Gift, Tao, \& Irwin, 2004). 
Clearly, college students represent an important young population segment for research and prevention of sexual risk behaviors. However, only a few national studies have addressed the sexual practices of college students. Among the first efforts was the 1995 National College Health Risk Behavior Survey (Douglas et al., 1997), in which $86.1 \%$ of students reported being sexually experienced and $34.5 \%$ having six or more sexual partners in their lifetimes; among students who were sexually active during the past month, only $27.9 \%$ indicated using condoms "almost or most of the time." This 1995 national survey has not been repeated since then. In a more recent study, the National College Health Assessment (American College Health Association, 2007), 52.1\% of students reported condom use during their last vaginal intercourse and only $27.7 \%$ reported using condoms during their last anal intercourse. These results are particularly alarming in light of the high rates of new STD/ HIV infections each year among youth. These findings also demonstrate the need to further investigate and to reduce sexual risk-taking behaviors in the college population.

\section{Heavy Episodic Drinking and Sexual Risk-Taking Among College Students}

To better understand risky sexual behaviors among college students, one should take into consideration that college years are generally associated with a multitude of other risk behaviors (Douglas et al., 1997). For example, approximately two in five college students engage in "binge drinking" (also referred to as heavy episodic drinking, HED), and given its pervasiveness on college campuses and its associated negative health and social consequences, HED has been a major concern to the college community (DeJong \& Langford, 2002; Wechsler, Davenport, Dowdall, Moeykens, \& Castillo, 1994; Wechsler et al., 2002). Of particular interest and relevance to the current study is that alcohol use, especially HED, has also been documented in numerous studies to be related to unplanned/unprotected sex and having multiple sex partners in the adolescent and college student populations (Halpern-Felsher, Millstein, \& Ellen, 1996; Hingson, Heeren, Winter, \& Wechsler, 2003; Wechsler, Dowdall, Davenport, \& Castillo, 1995).

\section{Problem Gambling and Sexual Risk Behaviors}

In contrast to college student drinking, problem gambling has been relatively understudied, but this risk behavior has increasingly received more research attention as legalized gambling expands throughout North America and emerging evidence suggests that youth represent one of the highest-risk population segments for gambling problems (Huang \& Boyer, 2007; Jacobs, 2000; National Research Council, 1999). Notably, using meta-analysis to synthesize previous gambling research in the U.S. and Canada, Shaffer and Hall (2001) estimated that the lifetime prevalence of Level 2 (sub-clinical/at-risk) and
Level 3 (clinical/pathological) gambling among college students was $10.9 \%$ and $5.6 \%$, respectively, more than double of that for adults ( $4.2 \%$ and $1.9 \%$, respectively). However, unlike the case with alcohol use, little is known about the potential linkage between problem gambling and sexual risk-taking. One of the earliest known studies to evaluate sexual risk behaviors in relation to problem gambling reported that, among a sample of substance abusers, those with gambling problems engaged in more HIV sexual risk behaviors than did those without gambling problems (Petry, 2000). A more recent study (Martins, Tavares, da Silva Lobo, Galetti, \& Gentil, 2004) also found increased sexual risk behaviors among pathological gamblers exhibiting elevated impulsivity.

\section{The Current Study}

Petry's (2000) pioneering study in substance abusers on the association between problem gambling and increased sexual risk behaviors suggested that future research should separate the effects of problem gambling and substance use on sexual risktaking. To address this issue, the study sample needs to include problem gamblers with and without substance use problems as in the present study. Based on the risk behavior literature reviewed above, college students are at high risk for gambling problems. College athletes are also a high-risk group for heavy alcohol use problems (Huang, DeJong, Schneider, \& Towvim, 2006; Wechsler, Davenport, Dowdall, Grossman, \& Zanakos, 1997). Taken together, there is reason to be concerned that college athletes may be susceptible to elevated sexual risktaking. However, to our knowledge, no studies in the published literature have examined sexual risk-taking in connection with both problem gambling and heavy drinking behaviors in the college athlete population. Hence, the main objective of this study was to fill this gap in knowledge by providing empirical evidence of the putative relationships among these risk behaviors. Specifically, using data from a national college athlete survey, this study examined the prevalence patterns of sexual risk-taking behaviors (i.e., unprotected sex and having multiple sex partners) in relation to levels of gambling problems and HED status among U.S. college athletes. To supplement the above comparisons, multivariate logistic regression was also used to evaluate and disentangle the effects of problem gambling and HED on sexual risk-taking. In addition, data were analyzed and reported separately by gender throughout this study to account for the effect of gender.

\section{Method}

Participants

Data from a representative national sample of 20,739 U.S. college athletes were derived from the National Collegiate 
Athletic Association (NCAA) national survey of problem gambling and health-risk behaviors. The response rate was between 65 and 75\%. Males (approximately 62\%) were slightly overrepresented in this sample, compared with the full NCAA student-athlete population ( $58 \%$ males; $42 \%$ females). In regard to age, $88.8 \%$ of this college athlete sample was $18-21,10.8 \%$ was $\geq 22$, and $0.4 \%$ was under 18 . Among participants reporting their race/ethnicity, $75 \%$ described themselves as white, $15 \%$ as black, and $10 \%$ as from another racial/ethnic group. These proportions approximate those seen in the overall population of U.S. college athletes (National Collegiate Athletic Association, 2004). Since the present study examined sexual risk behaviors among college athletes, we only included sexually active participants for analyses. Accordingly, 9,108 males and 4,762 females remained in the analysis of unprotected sex; 9,127 males and 4,767 females remained in the analysis of multiple sex partners.

\section{Measures}

There were two questions pertaining to unprotected sex and multiple sex partners. As regards unprotected sex, participants were asked, "Within the past year, have you, or have your partner(s), used a condom during sexual relations?" Response categories included: (1) I did not have sexual relations; (2) used all the time; (3) used most of the time; (4) used some of the time; and (5) never used a condom. Regarding multiple sex partners, participants were asked, "With how many different partners have you had sex during the past year?" Response categories included none, one, two, three or four or more. In addition, the survey also asked the participants: "Have you been in a committed sexual relationship during the past year? (Yes/No)" The committed sexual relationship status was included as an integral sexual risk component in our decision rule on classification. Hence, taking into account the stability of the sexual relationship in our measures of sexual risk behaviors, participants were classified as having had unprotected sex if they indicated ever having sex without using a condom and if they had not been in a committed sexual relationship during the past year. Similarly, participants were classified as having multiple sex partners if they reported having had sex with two or more different partners and if they had not been in a committed sexual relationship during the past year. With regard to HED status, participants were classified according to whether they ever had five or more drinks of alcohol at one sitting during the past month.

Problem gambling was assessed using the DSM-IV (American Psychiatric Association, 1994) Gambling Screen, which consists of 10 questions corresponding with the 10 diagnostic criteria associated with gambling problems (e.g., "During the past year, have you needed to gamble with larger amounts of money or with larger bets in order to obtain the same feeling of excitement?"). Responses to the 10 items were summed to create a score, ranging from 0 to 10 , with a higher score indicating more gambling problems. The 10 DSM diagnostic criteria have been found to exhibit satisfactory reliability, validity, and classification accuracy (Stinchfield, Govoni, \& Frisch, 2005). Accordingly, gamblers were classified as social gamblers if they had a DSM score of 0-2, and gamblers were classified as problem gamblers if they had a DSM score of 3-10. It is noteworthy that, in a clinical setting, a DSM score of $\geq 5$ typically indicates pathological gambling. However, given that this was a population-based study and that the sample size of gamblers who met the clinical pathological criteria was smaller than ideal for cross-group statistical analyses, all gamblers with a $D S M$ score of $\geq 3$ were classified as problem gamblers to represent those who experienced at least a somewhat problematic level of disordered gambling.

Procedure

The survey was conducted in consultation with the Director of Athletics and with the assistance of the Faculty Athletics Representative (FAR) at each participating school. The FAR was provided with a specific protocol to follow and script to read, which emphasized that the study was completely voluntary, that each student's responses were anonymous, and that voluntary completion of this study constituted the informed consent to participate, as reiterated on the survey form. The FAR distributed the survey to all athletes of a sampled team on the same occasion. The last team member to complete the survey was asked to seal and send the pre-addressed, prepaid envelope with completed surveys to the NCAA.

\section{Results}

\section{Unprotected Sex}

Table 1 shows the percentage of college athletes who reported engaging in unprotected sex during the past year, by gender, age, gambling, and drinking variables. Overall, male athletes $(10.2 \%)$ reported significantly higher prevalence of unprotected sex than female athletes $(7.9 \%)$. When examined by age, males $\geq 22$ had significantly higher prevalence of unprotected sex $(14.3 \%)$ than younger males $(9.4 \%)$; the age comparison was not significant in females. Male problem and social gamblers both appeared to have lower prevalence of unprotected sex than male non-gamblers, but the comparison was not significant ( $p=.051$ ). By contrast, female problem gamblers $(21.4 \%)$ reported significantly higher prevalence of unprotected sex than female social gamblers (8.8\%) and nongamblers $(7.3 \%)$. In regard to drinking, both male $(10.7 \%)$ and female $(9.2 \%)$ HED drinkers reported significantly higher prevalence of unprotected sex than their counterparts. 
Table 1 Percentage of college athletes who reported unprotected sex, by gender, age, gambling, and drinking variables

HED Heavy episodic drinking

* Chi-square comparison by gender $(10.2 \%$ vs. $7.9 \%)$ : $\chi^{2}(d f=1)=19.52, p<.001$

Table 2 Percentage of college athletes who reported multiple sex partners, by gender, age, gambling, and drinking variables

HED Heavy episodic drinking

* Chi-square comparison by gender (14.6\% vs. $9.3 \%)$ : $\chi^{2}(d f=1)=77.45, p<.001$

\section{Multiple Sex Partners}

The percentage of college athletes who reported having multiple sex partners during the past year is presented in Table 2, by gender, age, gambling, and drinking variables. The patterns were similar to those of unprotected sex, with males (14.6\%) having significantly higher prevalence of multiple sex partners than females $(9.3 \%)$. Males $\geq 22$ also had significantly higher prevalence of multiple sex partners $(17.9 \%)$ than younger males (13.9\%); the age comparison was not significant among females. When examined across gambling levels, the prevalence of multiple sex partners significantly decreased from $16.0 \%$ among male non-gamblers to $13.7 \%$ and $12.1 \%$ among male social and problem gamblers, respectively. Although female problem gamblers reported higher prevalence of multiple sex partners $(26.7 \%)$ than their counterparts, the comparison was not significant $(p=.068)$. As regards heavy drinking, both male $(15.0 \%)$ and female (10.8\%) HED drinkers reported higher prevalence of multiple sex partners than their counterparts; however, the comparison was not significant among males $(p=.093)$.

\section{Multivariate Logistic Regression Models}

Table 3 presents two multivariate logistic regression models estimating the effects of age, gambling, and drinking variables on unprotected sex among college athletes, separately by gender. The model for male athletes indicated that, controlling for the effects of gambling and drinking, males $\geq 22$ were $66 \%$ more likely than younger males to engage in unprotected sex $(\mathrm{OR}=1.66,95 \% \mathrm{CI}=1.38-2.00)$. Furthermore, male social gamblers were $22 \%$ less likely than male non-gamblers 
Table 3 Multivariate logistic regression models estimating the effects of age, gambling, and drinking variables on unprotected sex among college athletes, separately by gender

$\beta$ Logit coefficient, Adj. OR Adjusted odds ratio, $C I$ Confidence interval Ref: Reference category Bold: $p<.05$

\begin{tabular}{|c|c|c|c|c|c|c|}
\hline \multirow{2}{*}{ Variable } & \multicolumn{3}{|c|}{ Males } & \multicolumn{3}{|c|}{ Females } \\
\hline & $\beta$ & Adj. OR & $(95 \% \mathrm{CI})$ & $\beta$ & Adj. OR & $(95 \% \mathrm{CI})$ \\
\hline \multicolumn{7}{|l|}{ Age } \\
\hline$<22$ (ref) & .00 & 1.00 & & .00 & 1.00 & \\
\hline$\geq 22$ & .51 & 1.66 & $(1.38,2.00)$ & .20 & 1.22 & $(0.81,1.85)$ \\
\hline \multicolumn{7}{|l|}{ Gambling } \\
\hline Non-gambler (ref) & .00 & 1.00 & & .00 & 1.00 & \\
\hline Social gambler & -.25 & 0.78 & $(0.66,0.91)$ & .13 & 1.13 & $(0.88,1.46)$ \\
\hline Problem gambler & -.31 & 0.74 & $(0.50,1.09)$ & 1.25 & 3.50 & $(1.02,12.06)$ \\
\hline \multicolumn{7}{|l|}{ Drinking } \\
\hline Non-drinker (ref) & .00 & 1.00 & & .00 & 1.00 & \\
\hline Non-HED drinker & -.11 & 0.90 & $(0.63,1.28)$ & .51 & 1.66 & $(0.94,2.93)$ \\
\hline HED drinker & .31 & 1.37 & $(1.09,1.72)$ & 1.03 & 2.80 & $(1.70,4.59)$ \\
\hline
\end{tabular}

$(\mathrm{OR}=0.78,95 \% \mathrm{CI}=0.66-0.91)$ and, male HED drinkers were $37 \%$ more likely than male non-drinkers $(\mathrm{OR}=1.37$, $95 \% \mathrm{CI}=1.09-1.72$ ), to engage in unprotected sex. By contrast, the model for female athletes revealed that, while the effect of age was not significant, female problem gamblers were 3.5 times as likely as female non-gamblers (OR $=3.50,95 \%$ $\mathrm{CI}=1.02-12.06)$ and, female HED drinkers were 2.8 times as likely as female non-drinkers $(\mathrm{OR}=2.80,95 \% \mathrm{CI}=1.70$ 4.59), to engage in unprotected sex.

Similarly, Table 4 shows another two multivariate logistic regression models estimating the effects of age, gambling, and drinking variables on multiple sex partners among college athletes, separately by gender. Again, controlling for the effects of gambling and drinking, the model for male athletes also found that males $\geq 22$ were $29 \%$ more likely than younger males to have multiple sex partners $(\mathrm{OR}=1.29,95 \% \mathrm{CI}=$ 1.09-1.53). Moreover, male social and problem gamblers were $18 \%$ and $30 \%$ less likely than male non-gamblers $(\mathrm{OR}=$ $0.82,95 \% \mathrm{CI}=0.71-0.94$, and $\mathrm{OR}=0.70,95 \% \mathrm{CI}=0.49$ 0.98 , respectively) to have multiple sex partners; the effect of HED was not significant. Finally, the model for female athletes indicated that the effects of age and gambling on multiple sex partners were not significant. However, female HED drinkers were 2.11 times as likely as female non-drinkers to have multiple sex partners $(\mathrm{OR}=2.11,95 \% \mathrm{CI}=1.41-3.16)$.

\section{Discussion}

\section{Gender Differences in Sexual Risk-Taking Patterns}

Overall, males reported higher prevalence of unprotected sex and multiple sex partners than females in this college athlete sample. It is probable that, as has been found in the general college student population, males scored higher than females on sensation-seeking and thus were more likely to engage in risk-taking behaviors, including low condom use and more sex partners (Arnold, Fletcher, \& Farrow, 2002; Wagner, 2001). Among male athletes, an inverse relationship was found between gambling levels and the odds of sexual risktaking. The seemingly "protective" effect of gambling suggests that, as opposed to sexual risk behaviors, gambling may
Table 4 Multivariate logistic regression models estimating the effects of age, gambling, and drinking variables on multiple sex partners among college athletes, separately by gender

$\beta$ Logit coefficient, Adj. OR Adjusted odds ratio, $C I$ Confidence interval Ref: Reference category Bold: $p<.05$

\begin{tabular}{|c|c|c|c|c|c|c|}
\hline \multirow[t]{2}{*}{ Variable } & \multicolumn{3}{|l|}{ Males } & \multicolumn{3}{|c|}{ Females } \\
\hline & $\beta$ & Adj. OR & $(95 \% \mathrm{CI})$ & $\beta$ & Adj. OR & $(95 \% \mathrm{CI})$ \\
\hline \multicolumn{7}{|l|}{ Age } \\
\hline$<22$ (ref) & .00 & 1.00 & & .00 & 1.00 & \\
\hline$\geq 22$ & .25 & 1.29 & $(1.09,1.53)$ & .25 & 1.29 & $(0.88,1.88)$ \\
\hline \multicolumn{7}{|l|}{ Gambling } \\
\hline Non-gambler (ref) & .00 & 1.00 & & .00 & 1.00 & \\
\hline Social gambler & -.20 & 0.82 & $(0.71,0.94)$ & -.03 & 0.97 & $(0.76,1.23)$ \\
\hline Problem gambler & -.36 & 0.70 & $(0.49,0.98)$ & 1.08 & 2.95 & $(0.88,9.94)$ \\
\hline \multicolumn{7}{|l|}{ Drinking } \\
\hline Non-drinker (ref) & .00 & 1.00 & & .00 & 1.00 & \\
\hline Non-HED drinker & .07 & 1.08 & $(0.81,1.42)$ & .22 & 1.24 & $(0.77,2.00)$ \\
\hline HED drinker & .17 & 1.18 & $(0.98,1.44)$ & .75 & 2.11 & $(1.41,3.16)$ \\
\hline
\end{tabular}


be a competing risk behavior among these male college athletes. In other words, being excessively preoccupied with gambling is a common characteristic of problem gamblers and, as gambling severity increases, problem gamblers may become more socially isolated and immersed in gambling activities. As such, they are more likely to spend their time gambling, and not looking for sex outside their committed sexual relationships, if any, thereby lowering the odds of their sexual risk-taking.

By contrast, female problem gamblers in this study were 3.5 times as likely as female non-gamblers to engage in unprotected sex. A possible explanation may be related to the financial difficulties among problem gamblers as discussed in Petry's (2000) report. That is, these female problem gamblers may be more likely to engage in risky sexual acts with casual sex partners in exchange for money to finance their gambling or pay their debts. Another explanation is that these problem gamblers may be greater sensation-seekers and risk-takers than social gamblers and non-gamblers (Kuley \& Jacobs, 1988; Powell, Hardoon, Derevensky, \& Gupta, 1999). Hence, they may seek more stimulation not only via gambling but also through novel risky sexual experiences as well. Further, there has been research suggesting a positive relationship between irrational thinking regarding chance of outcome and risk-taking among gamblers (Delfabbro $\&$ Winefield, 2000). Previous research has also indicated that, people with optimistic biases feel that they are less likely than their peers to be affected by disease or danger, and therefore less likely to adopt risk-reducing precautions (Weinstein, 1989). Moreover, it has been documented in a college student study that females reported higher optimistic bias scores than males regarding their risk of contracting HIV/AIDS (Arnold et al., 2002). Put together, erroneous gambling expectancies about chance and unrealistic optimistic biases may lead to distorted sexual risk perceptions and, consequently, greater sexual risk-taking among these female problem gamblers.

As regards the effect of heavy drinking, while both male and female HED athletes reported elevated sexual risk behaviors, compared with their non-drinking peers, the effect of HED on sexual risk-taking was twice as large in females as in males. This suggests that HED plays a relatively more important role in sexual risk-taking among these female athletes than among their male peers. Findings from prior studies on sensation-seeking, alcohol expectancies, and risktaking may provide some insight into why HED athletes engaged in more sexual risk behaviors than their non-HED peers. For example, a recent study found that female college students who were high sensation-seekers tended to engage in HED, perceive less danger while consuming alcohol, and take more sexual risks, including unprotected sex with multiple sex partners (Gullette \& Lyons, 2006). Moreover, path analyses of a study also showed that sensation-seeking was associated with stronger expectancies that alcohol use could enhance sex, which then contributed to alcohol use in sexual contexts that was subsequently related to unprotected sex (Kalichman, Cain, Zweben, \& Swain, 2003). Therefore, it is reasonable to suggest that, compared with non-HED peers, HED athletes may be greater sensation-seekers and may have stronger alcohol expectancies regarding sexual enhancement, and hence were more likely to engage in sexual risktaking behaviors, as consistently found in this study.

Lastly, results of our study revealed that older male athletes were more likely than younger males to engage in sexual risk behaviors, suggesting that as these male athletes grew older, they might also become more sexually experienced and risk-taking. While older female athletes also appeared to have increased prevalence of sexual risk behaviors, the effect was not significant.

\section{Interaction Effects by Gender}

In view of the above differences between male and female athletes in their patterns of sexual risk-taking across gambling and drinking behaviors, interaction effects by gender were tested. For unprotected sex, the interaction effects of social gambling and problem gambling by gender were both found to be significant, with male social gamblers and problem gamblers being significantly less likely than their female counterparts to engage in unprotected sex. Such gender differences are particularly apparent when looking at the opposite directions of the observed effects of gambling on unprotected sex, as shown in the regression models for males versus females. Further, the interaction effect of HED by gender was also significant, indicating that, while male HED drinkers were more likely to engage in unprotected sex than male non-drinkers, male HED drinkers were less likely to engage in unprotected sex when compared with female HED drinkers. Similarly, for multiple sex partners, the interaction effects of problem gambling and HED by gender were both significant, with the same patterns as for unprotected sex.

\section{Overlap Between Unprotected Sex and Multiple Sex Partners}

Given the risky sexual nature, it is reasonable to speculate that an overlapping group of risk-takers might engage in both sexual risk behaviors, accounting for the comparable prevalence patterns of unprotected sex and multiple sex partners observed in this study. An ancillary analysis (data not shown) was conducted, accordingly, and revealed that, among male athletes who reported unprotected sex or multiple sex partners, $40.7 \%$ reported engaging in both sexual risk behaviors, signifying an overlap of the sub-populations that engaged in these risk-taking behaviors; a similar overlap $(40.9 \%)$ was found among female athletes. Of great concern is that, both male and female athletes who reported having multiple sex partners in the past year were also more likely to report 
engaging in unprotected sex ( $49.2 \%$ and $53.5 \%$, respectively) than were their counterparts who had only one sex partner ( $3.5 \%$ and $3.2 \%$, respectively), $\chi^{2}(d f=1)=2,571.82$ and $1,409.55$, respectively, both $p \mathrm{~s}<.001$. The combination of both risky sexual behaviors-having multiple sex partners and more prevalent unprotected sex-further elevates the risk of STD/HIV infections among these college athletes and their partners. Clearly, those identified as having multiple sex partners represent a high-risk group that warrants more attention.

\section{Limitations and Future Directions}

This study had some limitations that may be addressed in future research. First, the survey did not collect information on participants' sexual orientation. According to a recent study in the general college population, students having same-sex sexual experiences were more likely to report multiple sex partners and less consistent condom use, compared with their exclusively heterosexual counterparts (Eisenberg, 2001). Hence, it would be interesting to examine whether such patterns replicate in the college athlete population. In addition, future research may also evaluate whether the observed relationships in this study among problem gambling, HED, and sexual risk-taking behaviors may differ between students with same-sex and opposite-sex experiences.

Another potential limitation of the study pertains to participants' interpretations of sexual relations. Since different sexual activities involve different levels of risk, the lack of definition of sexual relations in this survey may raise some concerns. However, there has been considerable consensus that adolescents and college students primarily associate "having sex" with two major sexual interactions (i.e., penile-vaginal and penile-anal intercourse) (Bersamin, Fisher, Walker, Hill, \& Grube, 2007; Pitts \& Rahman, 2001; Sanders \& Reinisch, 1999). These two sexual activities, when engaged in without use of condoms, could expose participants to substantially high risk for STD/ $\mathrm{HIV}$ infections and, as such, were also the target risk behaviors of this study. Given the above-noted empirical consensus among youth about what behaviors constitute "having sex," it is reasonable to believe that our sexual behavior variables (i.e., unprotected sex and multiple sex partners) tapped into and captured the two target sexual interactions involving penile penetrative intercourse. Nevertheless, to avoid any possible confusion, future studies will benefit from greater behavioral specificity when inquiring about sexual activities.

As discussed earlier, sensation-seeking appears to be a common personality trait that can help explain some of the observed links among problem gambling, HED, and sexual risk-taking. Hence, drawing on the associations demonstrated in this study, future research may include standardized measures of sensationseeking and empirically assess their relationships with these risk behaviors. In particular, more research is needed to reexamine the gender differences in sexual risk-taking patterns in relation to problem gambling. Further, considering that various types of gamblers and drinkers may have distinctly different expectancies which may account for motivational and attitudinal differences in risk-taking, future qualitative research is warranted to revisit and further explore these expectancies and to elucidate how some of the gambling and alcohol expectancies may affect individuals' decisions to take sexual risks. Pragmatically, erroneous beliefs and misperceptions regarding peer norms identified through this line of research can also inform intervention programs, such as social norms campaigns (Scholly, Katz, Gascoigne, \& Holck, 2005), to reduce risk behaviors.

A final issue with this study is its cross-sectional design, which constrained our ability to make causal inferences. Future longitudinal research is needed to ascertain the development and temporal relations of these seemingly co-occurring risk behaviors. However, regardless of the specific causal relationships, the fact that this study found empirical evidence of significant associations among these risk behaviors underscores the importance of raising awareness among college personnel, healthcare professionals, prevention workers, and researchers about the interrelationships of these risk behaviors. Youth gambling, in particular, is a relatively new area of study and, as such, the observed relationships between gambling and sexual risk-taking provide promising new directions for future research in both fields of gambling and sexual behaviors. Problem gambling also adds a new facet to the multitude of youth problem behaviors (Donovan \& Jessor, 1985). In light of the empirical interrelationships among problem gambling, HED, and sexual risk-taking, multi-component prevention programs are needed to address these risk behaviors simultaneously to achieve optimal effectiveness. For example, sexual health education and drinking prevention initiatives on college campuses may incorporate gambling issues as a program component. On a practical level, considering the observed increase in sexual risk-taking among female problem gamblers, female students may be screened for problem gambling as an alternative approach to identify sexual risk-takers and, accordingly, sexual health interventions may strategically target female students exhibiting signs of gambling problems.

STDs continue to be a major public health concern among youth in the U.S. as they are disproportionately affected by new infections each year. Despite all the research and intervention efforts, the rates for new diagnoses of HIV/AIDS among American youth did not decrease but remained relatively constant throughout the 1990s and into the twenty-first century (Biddlecom, 2004). Clearly, innovative approaches are needed to tackle this long-standing problem and persistent trend. Albeit with some limitations, this exploratory study provided empirical evidence of associations among these risk behaviors and suggested new directions for future research. To our knowledge, this study may be the first to use data from a large national sample of college athletes to evaluate sexual risk-taking behaviors in relation to problem gambling severity, 
while accounting for HED status. Hence, the prevalence estimates from this study could also serve as national baseline data for the monitoring of these risk behaviors in future cohorts of college athletes.

Acknowledgments The financial support for this study was provided by the National Collegiate Athletic Association (NCAA). The authors also thank the more than 20,000 college athletes for their close attention and candor in responding to the extensive questionnaire, representing the NCAA's first national study on collegiate gambling and associated health-risk behaviors.

\section{References}

American College Health Association. (2007). American College Health Association National College Health Assessment Spring 2006 Reference Group data report (abridged). Journal of American College Health, 55, 195-206.

American Psychiatric Association. (1994). Diagnostic and statistical manual of mental disorders (4th ed.). Washington, DC: Author.

Arnold, P., Fletcher, S., \& Farrow, R. (2002). Condom use and psychological sensation seeking by college students. Sexual and Relationship Therapy, 17, 355-366.

Bersamin, M. M., Fisher, D. A., Walker, S., Hill, D. L., \& Grube, J. W. (2007). Defining virginity and abstinence: Adolescents' interpretations of sexual behaviors. Journal of Adolescent Health, 41, 182-188.

Biddlecom, A. E. (2004). Trends in sexual behaviours and infections among young people in the United States. Sexually Transmitted Infections, 80(ii), 74-79.

Chesson, H. W., Blandford, J. M., Gift, T. L., Tao, G., \& Irwin, K. L. (2004). The estimated direct medical cost of sexually transmitted diseases among American youth, 2000. Perspectives on Sexual and Reproductive Health, 36, 11-19.

DeJong, W., \& Langford, L. M. (2002). A typology for campus-based alcohol prevention: Moving toward environmental management strategies. Journal of Studies on Alcohol, Supplement, 14, 140-147.

Delfabbro, P. H., \& Winefield, A. H. (2000). Predictors of irrational thinking in regular slot machine gamblers. Journal of Psychology, $134,117-128$.

Donovan, J. E., \& Jessor, R. (1985). Structure of problem behavior in adolescence and young adulthood. Journal of Consulting and Clinical Psychology, 53, 890-904.

Douglas, K. A., Collins, J. L., Warren, C., Kann, L., Gold, R., Clayton, S., et al. (1997). Results from the 1995 National College Health Risk Behavior Survey. Journal of American College Health, 46, 55-66.

Eisenberg, M. (2001). Differences in sexual risk behaviors between college students with same-sex and opposite-sex experience: Results from a national survey. Archives of Sexual Behavior, 30, 575-589.

Gullette, D. L., \& Lyons, M. A. (2006). Sensation seeking, self-esteem, and unprotected sex in college students. Journal of the Association of Nurses in AIDS Care, 17, 23-31.

Halpern-Felsher, B. L., Millstein, S. G., \& Ellen, J. M. (1996). Relationship of alcohol use and risky sexual behavior: A review and analysis of findings. Journal of Adolescent Health, 19, 331-336.

Hingson, R., Heeren, T., Winter, M. R., \& Wechsler, H. (2003). Early age of first drunkenness as a factor in college students' unplanned and unprotected sex attributable to drinking. Pediatrics, 111, 34-41.

Huang, J. H., \& Boyer, R. (2007). Epidemiology of youth gambling problems in Canada: A national prevalence study. Canadian Journal of Psychiatry, 52, 657-665.
Huang, J. H., DeJong, W., Schneider, S. K., \& Towvim, L. G. (2006). Measuring college student drinking: Illustrating the feasibility of a composite drinking scale. Substance Abuse, 27, 33-45.

Jacobs, D. F. (2000). Juvenile gambling in North America: An analysis of long term trends and future prospects. Journal of Gambling Studies, 16, 119-152.

Kalichman, S. C., Cain, D., Zweben, A., \& Swain, G. (2003). Sensation seeking, alcohol use and sexual risk behaviors among men receiving services at a clinic for sexually transmitted infections. Journal of Studies on Alcohol, 64, 564-569.

Kuley, N. B., \& Jacobs, D. F. (1988). The relationship between dissociative-like experiences and sensation seeking among social and problem gamblers. Journal of Gambling Behavior, 4, 197-207.

Martins, S. S., Tavares, H., da Silva Lobo, D. S., Galetti, A. M., \& Gentil, V. (2004). Pathological gambling, gender, and risk-taking behaviors. Addictive Behaviors, 29, 1231-1235.

National Collegiate Athletic Association. (2004). 1999-00-2002-03 NCAA Student-Athlete Ethnicity Report. Indianapolis, IN: Author.

National Research Council. (1999). Pathological gambling: A critical review. Washington, DC: National Academy Press.

Petry, N. M. (2000). Gambling problems in substance abusers are associated with increased sexual risk behaviors. Addiction, 95, 10891100 .

Pitts, M., \& Rahman, Q. (2001). Which behaviors constitute "having sex" among university students in the UK? Archives of Sexual Behavior, 30, 169-176.

Powell, J., Hardoon, K., Derevensky, J. L., \& Gupta, R. (1999). Gambling and risk-taking behavior among university students. Substance Use and Misuse, 34, 1167-1184.

Sanders, S. A., \& Reinisch, J. M. (1999). Would you say you "had sex" if...? Journal of the American Medical Association, 281, 275-277.

Scholly, K., Katz, A. R., Gascoigne, J., \& Holck, P. S. (2005). Using social norms theory to explain perceptions and sexual health behaviors of undergraduate college students: An exploratory study. Journal of American College Health, 53, 159-166.

Shaffer, H. J., \& Hall, M. N. (2001). Updating and refining prevalence estimates of disordered gambling behaviour in the United States and Canada. Canadian Journal of Public Health, 92, 168-172.

Stinchfield, R., Govoni, R., \& Frisch, G. R. (2005). DSM-IV diagnostic criteria for pathological gambling: Reliability, validity, and classification accuracy. American Journal on Addictions, 14, 73-82.

Wagner, M. K. (2001). Behavioral characteristics related to substance abuse and risk-taking, sensation-seeking, anxiety sensitivity, and self-reinforcement. Addictive Behaviors, 26, 115-120.

Wechsler, H., Davenport, A. E., Dowdall, G. W., Grossman, S. J., \& Zanakos, S. I. (1997). Binge drinking, tobacco, and illicit drug use and involvement in college athletics: A survey of students at 140 American colleges. Journal of American College Health, 45, 195-200.

Wechsler, H., Davenport, A., Dowdall, G., Moeykens, B., \& Castillo, S. (1994). Health and behavioral consequences of binge drinking in college: A national survey of students at 140 campuses. Journal of the American Medical Association, 272, 1672-1677.

Wechsler, H., Dowdall, G. W., Davenport, A., \& Castillo, S. (1995). Correlates of college student binge drinking. American Journal of Public Health, 85, 921-926.

Wechsler, H., Lee, J. E., Kuo, M., Seibring, M., Nelson, T. F., \& Lee, H. (2002). Trends in college binge drinking during a period of increased prevention efforts: Findings from 4 Harvard School of Public Health College Alcohol Study surveys: 1993-2001. Journal of American College Health, 50, 203-217.

Weinstein, N. D. (1989). Optimistic biases about personal risks. Science, 246, 1232-1233.

Weinstock, H., Berman, S., \& Cates, W. (2004). Sexually transmitted diseases among American youth: Incidence and prevalence estimates, 2000. Perspectives on Sexual and Reproductive Health, 36, 6-10. 